\title{
ANÁLISE ENTRE OS GASTOS DO GOVERNO LOCAL E O CRESCIMENTO ECONÔMICO DAS CIDADES PORTUÁRIAS
}

\author{
Analysis among local government expenditure and the economic growth \\ of port cities
}

Cristiane Aparecida da Silva

E-mail: cristianedasilva@ufgd.edu.br

Mestre em Contabilidade pela Universidade Federal de Santa Catarina; Contadora na Universidade

Federal da Grande Dourados.

Endereço para contato: Centro Socioeconômico, Campus Universitário, Trindade, 88040-970,

Florianópolis, Santa Catarina, Brasil.

https://orcid.org/0000-0003-4052-4231

Barbara Gonçalves Amaral

E-mail: baah.amaral@outlook.com

Mestre em Contabilidade pela Universidade Federal de Santa Catarina.

https://orcid.org/0000-0001-9015-3251

Marcielle Anzilago

E-mail: marcianzilago@gmail.com

Mestre em Contabilidade pela Universidade Federal do Paraná; Doutoranda em Contabilidade pela

Universidade Federal de Santa Catarina.

https://orcid.org/0000-0001-5412-0786

Rogério João Lunkes

E-mail: rogeriolunkes@hotmail.com

Pós-doutor em Contabilidade pela Universidad de Valëncia, Espanha; Doutor e Mestre em Engenharia da Produção pela Universidade Federal de Santa Catarina; Professor no Programa de Pós-graduação em Contabilidade da Universidade Federal de Santa Catarina. https://orcid.org/0000-0003-4232-5746 


\section{Resumo}

O estudo investigou a relação existente entre as variáveis que compõem os gastos públicos e o crescimento econômico das cidades portuárias brasileiras. A estratégia de pesquisa usada para atender ao objetivo estabelecido foi a análise de dados secundários. Quanto à abordagem do problema, a pesquisa se enquadrou como quantitativa, em que a amostra foi intencional e não probabilística, compreendendo 32 cidades portuárias brasileiras. A dimensão temporal aplicada à pesquisa foi em um recorte transversal, compreendendo os Gastos Públicos per capita de 2013 e o Produto Interno Bruto (PIB) per capita de 2015. Com a finalidade de analisar a regressão, foi utilizado o software estatístico Gretl, e a partir dos aspectos avaliados, constatou-se que os gastos com saúde e investimentos possuem relação direta com o PIB per capita das cidades portuárias. Dessa forma, conclui-se que os gastos observados no estudo influenciaram o crescimento econômico, analisados por meio da variável PIB per capita das cidades portuárias elencadas para o presente trabalho. Por fim, em observação aos resultados do modelo analisado, concluiu-se que os gastos públicos possuem um alto poder de explicação, haja vista que 76,8\% do PIB per capita das cidades portuárias é explicado pelas variáveis dos gastos públicos investigados.

Palavras-chave: Gastos públicos. Crescimento econômico. Cidades portuárias. Portos. Governo local.

\section{Abstract}

The study investigated the relationship between the variables that make up public spending and the economic growth of Brazilian port cities. The research strategy used to meet the established objective was analysis of secondary data. Regarding the problem approach, this was quantitative, in which the research sample was intentional and not probabilistic, comprising 32 brazilian port cities. The time dimension applied to the research was in a transversal cut, comprising the per capita Public Expenses of 2013 and the per capita GDP of 2015. In order to analyze the regression, Gretl statistical software was used, and from the evaluated aspects, it was verified health and investment expenditures are directly related to the per capita GDP of the port cities. Thus, it can be concluded that the costs observed in the study influenced the economic growth, analyzed by the GDP per capita variable of the port cities listed for the present study. Finally, in observing the results of the analyzed model, it was concluded that public spending has a high explanatory power, since $76.8 \%$ of the GDP per capita of the port cities is explained by public expenditure.

Keywords: Public expenditures. Economic growth. Port cities. Ports. Local Government.

\section{INTRODUÇÃO}

Os governos veem o crescimento econômico como uma meta desejada com o propósito de ser aumentada, independente dos mecanismos utilizados (Zanmaria \& Castilho, 2006), sendo que uma de suas funções é a alocação de recursos pelo provimento de bens públicos, a distribuição da renda de forma equitativa aos cidadãos e a estabilização econômica, por meio da política fiscal (Riani, 2002). Essas finalidades atingem e são perseguidas pelas regiões e microrregiões nos diversos setores da economia.

Nas cidades portuárias, o crescimento econômico tem expressiva associação com os portos marítimos (Van Niekerk, 2005; Monié \& Vidal, 2006; Acciaro, 2008; Guerrero, Selva, 
\& Medina, 2008; Giner-Fillol, Lunkes, Ripoll-Feliu, \& Rosa, 2013; Shan, Yu \& Lee, 2014; Tukan, Achmadi, \& Widjaja, 2015; Jouili \& Allouche, 2016; Song \& Mi, 2016; Lea, Choib, \& Alfaroc, 2017). As cidades portuárias são identificadas como cidades que possuem portos, os quais realizam a maior parte das importações e exportações, principalmente de cargas de um país. As atividades das cidades portuárias afetam a economia das cidades vizinhas (Shan et al., 2014; Tukan et al., 2015).

As operações portuárias colaboram com o aumento da receita fiscal e o crescimento econômico (Acciaro, 2008; Bottasso, Conti, Ferrari, Merk \& Tei, 2013; Shan et al., 2014; Tukan et al., 2015). Consequentemente, o governo das cidades portuárias procura incentivar o desenvolvimento dos portos, visando atrair negócios e fornecendo políticas favoráveis a investimentos regionais (Shan et al., 2014). Além dessa questão, existe uma inquietude sobre a alocação dos gastos públicos na economia, em razão, especialmente, do impacto que eles provocam no crescimento econômico.

$\mathrm{Na}$ literatura acerca do assunto existem diversos trabalhos teóricos e empíricos que tratam da relação existente entre os gastos públicos e o crescimento econômico, e entre esses estudos está o de Adolf Wagner (1883), considerado um dos primeiros economistas a tutelar essa relação. Para esse autor, o crescimento da renda per capita exige cada vez mais do governo a oferta de mais bens públicos (Wagner, 1883 citado em Chan, Ramly, \& Karim, 2017).

A interferência do governo mediante a eficiência dos gastos públicos nas diversas esferas administrativas contribui para ajustar o mercado e agenciar o crescimento econômico de longo prazo (Riani, 2002; Van Niekerk, 2005; Acciaro, 2008; Shan et al., 2014; Tukan et al., 2015; Song \& Mi, 2016; Chan et al., 2017).

$\mathrm{Na}$ acepção de Wagner (1883), o desenvolvimento e o crescimento econômico da população, a urbanização, a expansão da cultura e a distribuição de renda eram fatores que exigiam a cooperação cada vez mais acentuada do Estado, o qual detém participação progressiva, com vistas ao desenvolvimento efetivo da economia, ampliando a dependência entre gastos públicos e o desenvolvimento econômico (Riani, 2002).

No que diz respeito ao Brasil, elementos como a má-distribuição de renda, a precariedade da educação e da saúde públicas são situações capazes de fazer os cidadãos refletirem a respeito de como e em que os entes públicos vêm investindo seus recursos (Zanmaria \& Castilho, 2006). Dada a importância socioeconômica atribuída aos gastos públicos efetuados pelo governo, emerge a seguinte questão-problema: qual a relação entre os gastos públicos e o crescimento econômico nas cidades portuárias?

Este estudo objetiva investigar a relação entre os gastos públicos e o crescimento econômico nas cidades portuárias brasileiras. Para atender a tal objetivo, analisaram-se dados secundários divulgados em portais públicos, utilizando o software estatístico Gretl. 
O estudo justifica-se quanto à sua importância, originalidade e viabilidade (Castro, 1977). Com relação à sua importância, justifica-se por melhorar o nível de informações para os formuladores de políticas públicas, gestores públicos, administradores privados, e tornar transparente ao cidadão a relação dos gastos públicos e também o crescimento da economia nas cidades portuárias brasileiras, fornecendo subsídios para o exercício do controle social, bem como para fomentar mais estudos nessa área do conhecimento.

Quanto à originalidade, existe a carência de estudos empíricos dos gastos das cidades portuárias comparando-os com o crescimento econômico local. Quanto à sua viabilidade, por despertar o interesse dos pesquisadores na busca de conhecimento nesse tema e pela acessibilidade dos dados nos portais públicos.

Este artigo estrutura-se em cinco seções. Além desta introdução, a segunda seção apresenta a fundamentação teórica subdividida em gastos públicos, crescimento econômico e cidades portuárias; na terceira seção são demonstrados os procedimentos metodológicos e ela está subdividida em enquadramento metodológico e procedimentos para a coleta e análise de dados; a quarta seção destina-se à análise e à discussão dos resultados da pesquisa; a quinta seção tece as conclusões; e, por último, são expostas as referências.

\section{FUNDAMENTAÇÃO TEÓRICA}

\subsection{GASTOS PÚBLICOS}

Na acepção de Barro (1990), o comportamento dos gastos governamentais é o que define a sua relação com o Produto Interno Bruto (PIB) per capita. Dessa forma, o governo pode optar por gastar menos ou mais na alocação dos serviços públicos. A figura da política fiscal no crescimento econômico é uma discussão que há muito tempo vem ganhando espaço na literatura, cujo papel é relevante em decorrência da sua capacidade de incentivar direta ou indiretamente a acumulação dos determinantes do crescimento econômico.

As alterações nos níveis de gastos afetam o nível de renda do País (Brasil), pois o Governo pode expandir sua demanda agregada, injetando recursos no setor privado por meio da compra e bens e serviços ou diminuindo a sua tributação, como também pode contrair sua demanda, com diminuição de seus gastos, repercutindo na demanda por bens e serviços no setor privado ou elevando o nível de tributação (Bogoni, Hein, \& Beuren, 2011).

Os componentes dos gastos públicos e suas relações com o crescimento econômico vêm sendo discutidos na literatura, especificamente na esfera da economia pública (Landau, 1983; Hsieh \& Lai, 1994; Sinha, 1998; Al-Faris, 2002; Kolluri, Panik, \& Wahab, 2000; Oliveira, 
2004; Chen, 2006; Irmen \& Kuehnel, 2009; Samudram, Nair, \& Vaithilingam, 2009; Merza \& Alhasan, 2016; Morozumi \& Veiga, 2016; Ashwani \& Sheera, 2017; Lupu \& Asandului, 2017).

Esse interesse de investigar o financiamento público e o fenômeno de crescimento em uma perspectiva em longo prazo se deve ao impacto do investimento público na tomada de decisão privada (Kolluri et al., 2000; Samudram et al., 2009), por também querer averiguar a competência do governo para realocar recursos com a finalidade de atingir os objetivos econômicos almejados (Irmen \& Kuehnel, 2009; Chan et al., 2017).

A maioria dos estudos elencados constatou que o impacto dos gastos públicos no crescimento econômico trouxe resultados positivos e negativos, ou seja, foram inconclusivos (Samudram et al., 2009; Chan et al., 2017; Lupu \& Asandului, 2017). Esses gastos, enquanto forma de intervenção do governo, podem ser catalisadores econômicos se o Estado acrescer fundos para os gastos públicos de modo sistemático e não distorcido, a fim de retificar o mercado e, enfim, alavancar o crescimento econômico de longo prazo (Chan et al., 2017).

O investimento público para a formação de capital detém a capacidade de alterar o crescimento por meio de acréscimo da produção, redução de custo de transporte, tributação e investimento privado; à medida que os gastos de educação e saúde impulsionam a melhora da produtividade marginal do capital humano (Samudram et al., 2009; Morozumi \& Veiga, 2016; Ashwani \& Sheera, 2017; Lupu \& Asandului, 2017), ressalta-se que essa relação entre os gastos públicos e o crescimento econômico pode não derivar de condições econômicas favoráveis (Chan et al., 2017).

A relação é verificada mediante estudos de dados em painel entre os países, respeitando as características econômicas, políticas, sociais e culturais (Samudram et al., 2009; Ashwani \& Sheera, 2017), as influências da taxa de crescimento no PIB per capita, a participação dos gastos do governo e a relação entre o investimento privado e o PIB per capita (Landau, 1983; Hsieh \& Lai, 1994; Merza \& Alhasan, 2016; Lupu \& Asandului, 2017).

Na literatura, as principais teorias da Lei de Wagner (1883), a Escola Keynesiana e o modelo de crescimento endógeno de Barro (1990) buscam esclarecer a eficácia dos gastos governamentais com o crescimento econômico. A Lei de Wagner (1883) e a Escola Keynesiana enunciam a existência da causalidade entre gastos públicos e crescimento econômico (Sinha, 1998; Al-Faris, 2002; Kolluri et al., 2000; Samudram et al., 2009; Merza \& Alhasan, 2016; Chan et al., 2017; Lupu \& Asandului, 2017).

Para Wagner (1883), no processo de industrialização, como a renda per capita da nação aumenta, consequentemente a parcela dos gastos públicos também aumenta, refletindo a causalidade do crescimento econômico para os gastos públicos. Entretanto, na Escola Keynesiana, os gastos públicos são um elemento da política fiscal, utilizados como instrumento para induzir o crescimento, logo, a causalidade dos gastos públicos implica o crescimento econômico (Sinha, 1998; Samudram et al., 2009; Merza \& Alhasan, 2016). 
O modelo de crescimento endógeno de Barro (1990) explicita que o impacto dos gastos públicos acerca do crescimento econômico depende da sua estrutura e segrega as despesas públicas em produtivas ou improdutivas. As despesas públicas produtivas detêm o propósito de aumentar externamente a produção, e as despesas públicas de consumo servem para aumentar externamente a utilidade das famílias (Chen, 2006; Merza \& Alhasan, 2016; Lupu \& Asandului, 2017).

Na década de 1990, Oliveira (2004) realizou um estudo empírico acerca dos determinantes do crescimento econômico e populacional das cidades nordestinas brasileiras. Os resultados encontrados mostraram que o papel das transferências de renda pelo governo tem pouco ou quase nenhum efeito sobre o crescimento econômico e o crescimento populacional das cidades nordestinas. Por meio desses resultados, chegou-se à discussão a respeito do papel do governo local na promoção do crescimento econômico nas cidades.

Rajkumar e Swaroop (2008) pesquisaram em 91 países desenvolvidos e em desenvolvimento os vínculos entre gastos públicos, governança e resultados, bem como analisaram o papel da governança, medida pelo nível de corrupção e pela qualidade da burocracia na determinação da eficácia do gasto público e na melhoria dos resultados do desenvolvimento humano.

Os autores supramencionados descobriram que o diferencial do impacto do gasto público com educação e saúde depende da qualidade da governança, e concluíram que as despesas públicas em saúde e educação teriam maior probabilidade em países com governança aperfeiçoada.

Na pesquisa de Rajkumar e Swaroop (2008) a governança foi categorizada como fragilizada e ruim, os autores enfatizam que os gastos com saúde pública reduzem as taxas de mortalidade infantil, e, de maneira similar, os gastos públicos com o ensino primário tornam-se mais eficazes no aumento do sucesso do ensino nos países com boa governança.

\subsection{CRESCIMENTO ECONÔMICO}

A teoria econômica sugere que o crescimento do PIB é um componente importante do desenvolvimento econômico (Ogundari \& Awokuse, 2018). Nesse contexto, Bloom, Canning, e Sevilla (2004) argumentam que a educação adequada e a boa saúde estimulam uma força de trabalho mais produtiva que poderia, consequentemente, estimular o crescimento econômico. Ogundari e Abdulai (2014) ressaltam que uma força de trabalho mais instruída e mais saudável tem maior probabilidade de criar e adotar novas tecnologias, ampliando o PIB per capita e refletindo crescimento econômico. 
Nas palavras de Gyimah-Brempong e Wilson (2004), a proporção da renda nacional (receita fiscal) gasta em saúde tende a ser alta, superando $10 \%$ da receita total e aumenta nos países da Organização para a Cooperação e Desenvolvimento Econômico (OCDE). Já nos países menos desenvolvidos é inferior a 10\%, mas aumenta a um ritmo relativamente acelerado, embora nos países da África Subsaariana o ritmo tenha diminuído na última década.

No entanto, tanto Bloom et al. (2004) quanto Mayer (2001) enfatizam que os gastos públicos com saúde são relevantes e necessários, a qualidade da saúde na sociedade aumenta a produtividade da força de trabalho, reduzindo a incapacidade, a debilidade e o número de dias perdidos por doença, e, consequentemente, os cidadãos recebem melhores salários.

Thomas e Frankenberg (2002) enfatizam que indivíduos mais saudáveis têm maior expectativa de vida - o que estimula o crescimento econômico acelerando a transição demográfica (Ogundari \& Awokuse, 2018) - e, portanto, têm maiores incentivos para investir em treinamento e na aquisição de habilidades aprimoradas. No entanto, das medidas comumente usadas, a educação tem sido frequentemente vista como uma fonte importante para o crescimento econômico.

Isso porque, em uma economia do conhecimento, a educação desempenha o papel crucial de fornecer capital humano altamente qualificado necessário para a criação de empregos, crescimento econômico e prosperidade do indivíduo e da sociedade (Pegkas \& Tsamadias, 2014).

Estudos relacionando política fiscal e crescimento econômico foram desenvolvidos para a economia brasileira. Entretanto, o foco desses estudos se concentrou ou no impacto dos gastos agregados acerca do crescimento, ou no impacto somente dos investimentos em infraestrutura (e não nos efeitos diferenciados da composição dos gastos) com relação ao crescimento.

Tanto os estudos de Ferreira (1996) quanto os de Ferreira e Malliagros (1998) mostraram que o impacto dos investimentos em infraestrutura acerca do crescimento econômico brasileiro é positivo no longo prazo. Ferreira (1996) encontra esse resultado para o período de 1970-1993, considerando como variáveis gastos com investimento em: infraestrutura; telecomunicações; energia elétrica; portos; setor marítimo; e ferrovias. Já Ferreira e Malliagros (1998) analisaram o período de 1950 a 1995, considerando o gasto com investimento nos setores rodoviário e infraestrutura.

Bogoni et al. (2011) investigaram a relação entre o crescimento econômico, considerando - PIB per capita, e as variáveis que compõem os gastos públicos (gastos em saúde e saneamento, gastos municipais em educação e cultura, conjunto de investimentos no município, gastos com habitação e gastos com assistência e previdência) das maiores cidades da região Sul do Brasil. Com base nos resultados da pesquisa, os autores concluíram que é relevante o papel do governo local no provimento dos serviços públicos que afetam a produtividade e a qualidade de vida dos trabalhadores, e, consequentemente, o crescimento econômico. 
Merza e Alhasan (2016) examinaram a economia do Kuwait olhando especificamente para qual aspecto socioeconômico dos gastos do Governo tem maior efeito sobre o crescimento econômico. Por meio do teste de integração Johansen e do teste de causalidade de Granger, concluíram que os gastos com desenvolvimento têm demonstrado o maior impacto acerca da taxa de crescimento do PIB per capita, e os gastos com saúde e educação também apresentam relação de equilíbrio de longo prazo com o PIB per capita.

No entanto, os autores perceberam que os gastos não apresentam uma relação de causalidade entre si, e isso pode ser atribuído ao efeito mínimo de uma população de baixa renda quando comparada às rendas do petróleo e à possibilidade de má-gestão na dotação orçamental. Por meio dos achados, os autores perceberam um aumento nos gastos com projetos de desenvolvimento, especificamente relacionados à infraestrutura, e sugeriram reavaliar as alocações dos orçamentos com educação e saúde de uma maneira que aumentará a sustentabilidade e promoverá a eficiência global, levando em conta a demografia.

Chan et al. (2017) analisaram o impacto da eficiência do gasto do governo no crescimento econômico de 115 países com sistema de imposto acerca do valor agregado (IVA), e a partir dos aspectos observados os autores chegaram às seguintes conclusões: que a eficiência dos gastos do governo promove o crescimento econômico; o sistema IVA aumenta o efeito da eficiência dos gastos do governo acerca do crescimento econômico; e a qualidade da democracia e força do Legislativo aumentaria ainda mais o papel moderador do sistema IVA. Além disso, os pesquisadores ressaltaram que os resultados são robustos em ambos os países desenvolvidos e em desenvolvimento, porém, os melhores resultados estão nos países desenvolvidos.

\subsection{CIDADES PORTUÁRIAS}

As pesquisas evidenciam a importância dos portos e seu papel fundamental dentro da cadeia de abastecimento de produtos, que impulsionaram, ao longo do tempo, a reestruturação na logística e na gestão eficiente dos portos (Ensslin, Dutra, Ensslin, Valmorbida, \& Cardoso, 2015). O crescimento econômico nas cidades portuárias detém forte ligação com os portos marítimos, visto que o porto exerce atribuição de centro logístico ao promover o mercado global (Van Niekerk, 2005; Monié \& Vidal, 2006; Acciaro, 2008; Guerrero, Selva \& Medina, 2008; Giner-Fillol et al., 2013; Shan, Yu \& Lee, 2014; Tukan et al., 2015; Jouili \& Allouche, 2016; Song \& Mi, 2016; Lea, Choib, \& Alfaroc, 2017).

Nas palavras de Ensslin, Dezem, Dutra, e Ensslin (2017) o transporte marítimo desempenha um papel estratégico no comércio internacional, impactando as economias locais, regionais e globais, em razão do seu alcance, flexibilidade, mínimo impacto ambiental e 
custo-eficácia em termos de transporte de mercadorias. Na acepção de Ensslin et al. (2017) o transporte do oceano é o centro do palco, quando se trata de otimização de logística global. Essa posição privilegiada de transporte coloca pressão significativa em portos marítimos e sua gestão para melhorar o desempenho, de modo a influenciar a competitividade do transporte.

Nesse sentido, as operações portuárias proporcionam a entrada de informações, de recursos financeiros e de serviços que agregam valor às cidades portuárias (Van Niekerk, 2005; Shan et al., 2014; Tukan et al., 2015; Song \& Mi, 2016), e essas operações possibilitam indicadores de desenvolvimento e crescimento econômico (Giner-Fillol et al., 2013).

Muitos estudos salientam que as operações portuárias procederiam no acréscimo da receita fiscal e crescimento econômico acelerado como investimento e constituição de capital para a geração de renda regional (Acciaro, 2008; Bottasso et al., 2013; Shan et al., 2014; Tukan et al., 2015), apoiados pela Teoria Econômica em geral (Shan et al., 2014). Tal Teoria, em seus modelos teóricos, evidencia que o porto reduz o custo de transporte e atrai o desenvolvimento regional (Shan et al., 2014).

Essas operações portuárias impactam positivamente as cidades, tendo variações de impacto conforme o tamanho do porto, da região e dos tráfegos de cargas (Acciaro, 2008). Há estudos que analisam o impacto direto ou indireto dos portos nas cidades (Acciaro, 2008; Guerrero et al., 2008; Shan et al., 2014), associado ao impacto das atividades portuárias industriais e comerciais, turismo, emprego e renda. Desse modo, o porto marítimo beneficiase do crescimento econômico da cidade portuária, juntamente com os fatores demográficos e a infraestrutura (Monié \& Vidal, 2006).

O planejamento estratégico, o processo de implementação de política sustentável e desenvolvimento de infraestrutura, por meio de uma gestão eficiente, garantirão o desenvolvimento da cidade e sua sustentabilidade socioeconômica (Tukan et al., 2015). O Estado impulsiona o desenvolvimento portuário estabelecendo políticas favoráveis para atrair negócios ou investir na melhoria da infraestrutura (Van Niekerk, 2005; Acciaro, 2008; Shan et al., 2014; Tukan et al., 2015; Song \& Mi, 2016), em razão de que os efeitos de desenvolvimento portuário já instituem uma das principais justificativas para o investimento público (Acciaro, 2008).

\section{PROCEDIMENTOS METODOLÓGICOS}

\subsection{ENQUADRAMENTO METODOLÓGICO}

Como classificação dos objetivos da pesquisa, são de caráter descritivo, pois pretendem descrever as características de determinada população ou fenômeno, bem como 
o estabelecimento de relações entre variáveis e fatos (Cooper \& Schindler, 2011). Com relação aos procedimentos técnicos, classifica-se como análise de dados secundários, nesse caso, dados divulgados em portais públicos (Cooper \& Schindler, 2011).

Quanto à abordagem do problema, ela se enquadra como quantitativa na acepção de Richardson (1999), caracterizada pelo emprego de quantificação, tanto na modalidade de coleta de informações quanto no tratamento delas por meio de técnicas estatísticas.

\subsection{PROCEDIMENTOS PARA A COLETA DE DADOS}

Na Figura l, foi demonstrado o desenho da pesquisa, evidenciando que as operações portuárias contribuem com o acréscimo da receitais fiscais nas cidades, impulsionando os gastos públicos e, consequentemente, influenciando o crescimento econômico, como também aceleram o investimento e a constituição de capital para a geração de renda local e regional das cidades portuárias.

Figura 1

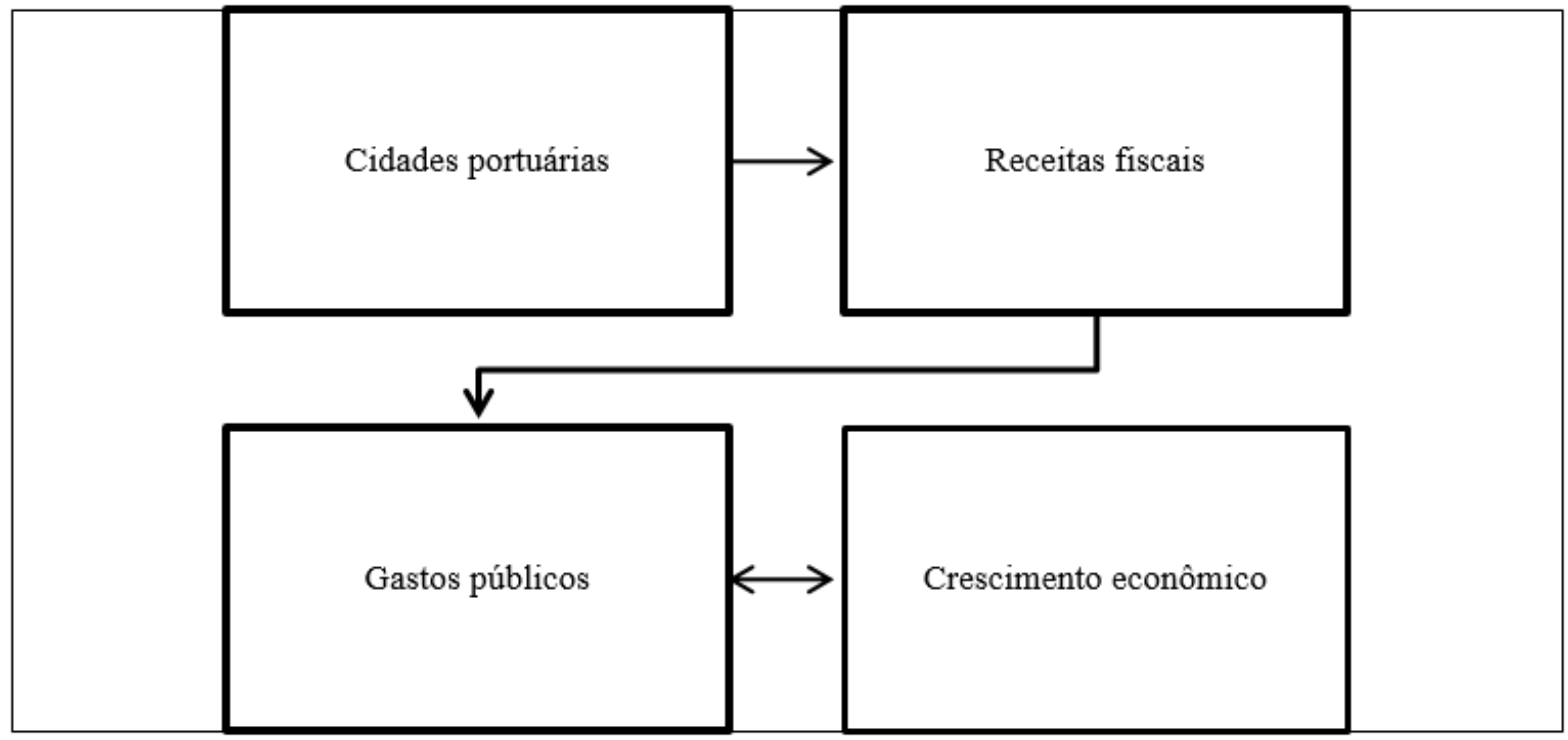

Desenho de pesquisa.

A população da pesquisa compreende as 32 cidades portuárias no Brasil, e a amostra da pesquisa é intencional e não probabilística, compreendendo essas cidades para a seleção da amostra, por meio de pesquisa realizada em junho de 2017 no site do Ministério dos Transportes, Portos e Aviação Civil, em que se coletou a lista de Portos Nacionais, por meio do Sistema Portuário Nacional.

Na sequência à coleta, examinou-se o site do Instituto Brasileiro de Geografia e Estatística, em que foram selecionadas essas cidades, em decorrência da presença de portos 
no seu limite territorial. As cidades selecionadas estão relacionadas em ordem de Estado, conforme demonstrado na Tabela 1:

Tabela 1

Cidades portuárias brasileiras

\begin{tabular}{llllll}
\hline Ordem & \multicolumn{1}{c}{ Cidade } & \multicolumn{1}{c}{ Estado } & Ordem & Cidade & Estado \\
\hline 1 & Maceió & Alagoas & 17 & Recife & Pernambuco \\
2 & Santana & Amapá & 18 & Angra dos Reis & Rio de Janeiro \\
3 & Candeias & Bahia & 19 & Niterói & Rio de Janeiro \\
4 & Ilhéus & Bahia & 20 & Rio de Janeiro & Rio de Janeiro \\
5 & Salvador & Bahia & 21 & São João da Barra & Rio de Janeiro \\
6 & Fortaleza & Ceará & 22 & Pelotas & Rio Grande do Sul \\
7 & São Gonçalo do Amarante & Ceará & 23 & Rio Grande & Rio Grande do Sul \\
8 & Anchieta & Espírito Santo & 24 & Imbituba & Santa Catarina \\
9 & Aracruz & Espírito Santo & 25 & Itajaí & Santa Catarina \\
10 & Vitória & Espírito Santo & 26 & Itapoá & Santa Catarina \\
11 & São Luís & Maranhão & 27 & Laguna & Santa Catarina \\
12 & Barcarena & Pará & 28 & Navegantes & Santa Catarina \\
13 & Belém & Pará & 29 & São Francisco do Sul & Santa Catarina \\
14 & João Pessoa & Paraíba & 30 & Santos & São Paulo \\
15 & Antonina & Paraná & 31 & São Sebastião & São Paulo \\
16 & Ipojuca & Pernambuco & 32 & Barra dos Coqueiros & Sergipe \\
\hline
\end{tabular}

Alicerçada na literatura pesquisada e com a finalidade de atingir o objetivo proposto, foram selecionados os construtos e variáveis para a realização desta pesquisa, conforme apresentado na Tabela 2, juntamente com as respectivas definições operacionais e fonte teórica:

Tabela 2

Construto de pesquisa

\begin{tabular}{|c|c|c|c|}
\hline Construto & Variáveis & Definição operacional & Fonte teórica \\
\hline $\begin{array}{l}\text { Crescimento } \\
\text { econômico }\end{array}$ & $\begin{array}{l}\mathrm{PIB} / \text { per } \\
\text { capita }\end{array}$ & $\begin{array}{l}\text { Produto Interno Bruto (soma de todos } \\
\text { os bens) dividido pela quantidade de } \\
\text { habitantes do município. }\end{array}$ & $\begin{array}{l}\text { Bogoni et al. (2011), Merza e Alhasan } \\
\text { (2016), Ogundari e Abdulai (2014), } \\
\text { Rajkumar e Swaroop (2008) }\end{array}$ \\
\hline \multirow{5}{*}{$\begin{array}{l}\text { Gastos } \\
\text { públicos }\end{array}$} & Cultura & $\begin{array}{l}\text { Gastos públicos dos governos locais - } \\
\text { referentes à Cultura }\end{array}$ & Bogoni et al. (2011) \\
\hline & Educação & $\begin{array}{l}\text { Gastos públicos dos governos locais - } \\
\text { referentes à Educação }\end{array}$ & $\begin{array}{l}\text { Bogoni et al. (2011), Merza e Alhasan } \\
\text { (2016), Ogundari e Abdulai (2014), } \\
\text { Rajkumar e Swaroop (2008) }\end{array}$ \\
\hline & Investimento & $\begin{array}{l}\text { Gastos públicos dos governos locais - } \\
\text { referentes a Investimento }\end{array}$ & $\begin{array}{l}\text { Bogoni et al. (2011), Ferreira (1996), } \\
\text { Ferreira e Malliagros (1998) }\end{array}$ \\
\hline & Saúde & $\begin{array}{l}\text { Gastos públicos dos governos locais - } \\
\text { referentes à Sáude }\end{array}$ & $\begin{array}{l}\text { Bogoni et al. (2011), Merza e Alhasan } \\
\text { (2016), Ogundari e Abdulai (2014), } \\
\text { Rajkumar e Swaroop (2008) }\end{array}$ \\
\hline & Urbanismo & $\begin{array}{l}\text { Gastos públicos dos governos locais - } \\
\text { referentes ao Urbanismo }\end{array}$ & Oliveira (2004) \\
\hline
\end{tabular}


Os índices do PIB per capita tiveram o ano 2015 como base, cuja coleta foi proveniente do site do Instituto Brasileiro de Geografia e Estatística ([IBGE], 2017). O PIB per capita é considerado um indicador econômico fundamental, pois atua na forma de subsídio e alicerce para os formadores de políticas econômicas, bem como para os tomadores de decisão tanto no âmbito governamental quanto no privado.

Os pesquisadores enfatizam que o PIB per capita é o resultado de todos os fatores e atividades da economia, ou seja, inclui-se o capital humano, o mercado financeiro, os imóveis e, ainda, qualquer mercado significativo (Furtuoso \& Guilhoto, 2003; Araújo, Fajardo, \& Tavani, 2006).

As variáveis de investimento, urbanismo e cultura justificam-se para a utilização pois representam os gastos produtivos, pois estes representam externalidades positivas, que podem ser absorvidas por uma parte significativa da economia local, impulsionando o crescimento econômico (Barro, 1990).

A variável do gasto com saúde nos modelos de crescimento endógeno seria ponderada como saúde preventiva, a qual influenciaria o crescimento econômico por garantir a permanência do cidadão no mercado de trabalho (Ashwani \& Sheera, 2017).

Na variável educação, considera-se que uma boa qualidade educacional minimizaria as disparidades que alteram o bem viver social, fornecendo mecanismo para a capacitação dos cidadãos e sua inserção no mercado, impactando no crescimento econômico (Zanmaria \& Castilho, 2006). Evidenciam-se na Tabela 3 os gastos públicos per capita das cidades que integram a amostra:

Tabela 3

Gastos públicos das cidades portuárias brasileiras referentes ao ano 2013

\begin{tabular}{|c|c|c|c|c|c|}
\hline Ordem & Saúde & Educação & Cultura & Urbanismo & Investimento \\
\hline Maceió & 478,90 & 214,78 & 6,04 & 16,45 & 16,32 \\
\hline Santana & 216,51 & 335,57 & 6,24 & 32,26 & 22,00 \\
\hline Candeias & 510,47 & 787,62 & 37,18 & 348,61 & $1.031,46$ \\
\hline Ilhéus & 311,76 & 420,77 & 0,76 & 29,86 & 9,71 \\
\hline Salvador & 307,92 & 264,31 & 1,41 & 201,11 & 1,99 \\
\hline Fortaleza & 546,71 & 298,40 & 13,90 & 228,15 & 130,19 \\
\hline São Gonçalo do Amarante & 516,93 & 994,66 & 35,17 & 73,70 & 215,46 \\
\hline Anchieta & $1.706,58$ & $2.481,66$ & 14,08 & $1.183,49$ & $3.459,30$ \\
\hline Aracruz & 547,48 & 989,76 & 11,75 & 448,71 & 362,66 \\
\hline Vitória & 694,12 & 886,14 & 37,56 & 475,95 & 18,87 \\
\hline São Luís & 562,86 & 418,38 & 18,02 & 85,88 & 53,22 \\
\hline Barcarena & 393,40 & 785,44 & 21,31 & 215,18 & 89,94 \\
\hline Belém & 438,04 & 276,81 & 8,48 & 274,34 & 125,64 \\
\hline João Pessoa & 709,75 & 419,66 & 19,91 & 134,86 & 78,31 \\
\hline
\end{tabular}




\begin{tabular}{|c|c|c|c|c|c|}
\hline Ordem & Saúde & Educação & Cultura & Urbanismo & Investimento \\
\hline Antonina & 456,35 & 432,05 & 32,85 & 277,72 & 50,53 \\
\hline Ipojuca & 961,24 & $1.420,02$ & 45,02 & 408,20 & 290,88 \\
\hline Recife & 454,52 & 396,21 & 52,03 & 454,83 & 331,95 \\
\hline Angra dos Reis & $1.157,98$ & 751,20 & 20,97 & 397,94 & 178,72 \\
\hline Niterói & 657,25 & 497,22 & 34,50 & 225,91 & $5.144,08$ \\
\hline Rio de Janeiro & 561,03 & 535,80 & 33,65 & 471,51 & 2,27 \\
\hline São João da Barra & $2.854,68$ & $1.824,30$ & 54,32 & $1.579,27$ & 266,89 \\
\hline Pelotas & 477,80 & 337,37 & 11,04 & 52,06 & 29,36 \\
\hline Rio Grande & 14,92 & 24,41 & 1,21 & 6,13 & 2,13 \\
\hline Imbituba & 439,15 & 470,26 & 10,45 & 119,80 & $1.856,50$ \\
\hline Itajaí & 834,18 & 881,34 & 37,16 & 435,45 & 183,42 \\
\hline Itapoá & 623,00 & 971,30 & 18,11 & 361,40 & 248,78 \\
\hline Laguna & 509,79 & 383,07 & 23,18 & 145,81 & 67,37 \\
\hline Navegantes & 369,85 & 798,20 & 27,29 & 318,75 & 74,11 \\
\hline São Francisco do Sul & 697,59 & 837,42 & 36,46 & 366,13 & 100,52 \\
\hline Santos & 816,99 & 756,33 & 64,55 & 520,60 & 182,84 \\
\hline São Sebastião & $1.356,67$ & $1.410,76$ & 171,35 & $1.039,68$ & 475,68 \\
\hline Barra dos Coqueiros & 421,28 & 558,53 & 19,83 & 235,25 & 64,18 \\
\hline
\end{tabular}

Na Tabela 3, a coleta dos valores acerca dos gastos públicos por cidades portuárias foi extraída de informações disponíveis no site da Secretaria do Tesouro Nacional ([STN], 2017), referentes ao ano 2013. Apresenta-se na Tabela 4 o PIB per capita concernente às cidades portuárias que compõem a amostra da pesquisa:

Tabela 4

Produto Interno Bruto per capita das cidades portuárias no ano 2015

\begin{tabular}{|c|c|c|c|c|c|}
\hline Ordem & Cidade & PIB per capita & Ordem & Cidade & PIB per capita \\
\hline 1 & Maceió & $20.400,62$ & 17 & Recife & $29.701,32$ \\
\hline 2 & Santana & $17.741,36$ & 18 & Angra dos Reis & $40.705,55$ \\
\hline 3 & Candeias & $38.121,89$ & 19 & Niterói & $51.779,62$ \\
\hline 4 & Ilhéus & $20.196,49$ & 20 & Rio de Janeiro & $49.527,98$ \\
\hline 5 & Salvador & $19.812,07$ & 21 & São João da Barra & $211.946,00$ \\
\hline 6 & Fortaleza & $22.092,58$ & 22 & Pelotas & $21.553,00$ \\
\hline 7 & São Gonçalo do Amarante & $39.143,02$ & 23 & Rio Grande & $34.997,50$ \\
\hline 8 & Anchieta & $99.873,80$ & 24 & Imbituba & $26.048,89$ \\
\hline 9 & Aracruz & $55.889,97$ & 25 & ltajaí & $91.856,35$ \\
\hline 10 & Vitória & $64.744,84$ & 26 & Itapoá & $30.214,42$ \\
\hline 11 & São Luís & $24.986,16$ & 27 & Laguna & $16.396,92$ \\
\hline 12 & Barcarena & $47.684,37$ & 28 & Navegantes & $45.000,91$ \\
\hline 13 & Belém & $20.340,21$ & 29 & São Francisco do Sul & $77.794,48$ \\
\hline 14 & João Pessoa & $23.169,14$ & 30 & Santos & $46.007,27$ \\
\hline 15 & Antonina & $19.789,87$ & 31 & São Sebastião & $44.470,05$ \\
\hline 16 & Ipojuca & $95.950,66$ & 32 & Barra dos Coqueiros & $41.168,50$ \\
\hline
\end{tabular}


Após a realização da coleta de dados das referidas cidades (Tabelas 3 e 4), estes foram organizados em planilha no software Microsoft Excel, e, em seguida, foi aplicada a regressão linear por meio da operacionalização do software estatístico Gretl, em que se analisaram os gastos per capita com saúde, educação, cultura, urbanismo e investimento, relacionando-os com o PIB per capita.

\section{RESULTADOS E DISCUSSÃO}

Esta seção contém a descrição e a análise dos dados coletados. Inicialmente, são apresentadas as estatísticas descritivas das variáveis que compõem os gastos públicos (saúde, educação, cultura e urbanismo e investimento) per capita e do PIB per capita, conforme se apresenta na Tabela 5, e posteriormente calculados estatisticamente por meio do software Gretl:

Tabela 5

Estatística descritiva

\begin{tabular}{lrrr}
\hline \multicolumn{1}{c}{ Variável } & Média & Mediana & \multicolumn{2}{c}{ Desvio padrão } \\
\hline Saúde & 675,18 & 531,82 & 514,88 \\
Educação & 714,37 & 547,16 & 506,23 \\
Cultura & 28,93 & 21,14 & 30,64 \\
Urbanismo & 348,91 & 276,03 & 344,53 \\
Investimento & 473,92 & 113,03 & $1.087,49$ \\
PIB per capita 2015 & $46.534,56$ & $38.632,46$ & $38.055,55$ \\
\hline
\end{tabular}

Observam-se na Tabela 5 a média, a mediana e o desvio padrão dos gastos per capita e PIB per capita das cidades portuárias. Conforme se observa na Tabela 5, os gastos públicos com saúde e educação nesses municípios são os maiores, $R \$ 675,18$ e $R \$ 714,37$, respectivamente, seguidos pelo investimento de $R \$ 473,92$, urbanismo com valor de $R \$ 348,91$ e cultura de $\mathrm{R} \$ 28,93$. Pode-se inferir que nas cidades portuárias há um maior investimento em saúde e educação, por fatores advindos das condições originadas nas respectivas cidades portuárias.

É possível perceber também PIB per capita em média elevada de R\$ 46.534,56. Quanto ao desvio padrão amostral, percebe-se a maior variabilidade em relação à média amostral dos gastos: a maior variabilidade foi encontrada nos gastos com investimento ( $R \$$ $38.055,55)$, seguidos por saúde ( $R \$ 514,88)$, educação ( $R \$ 506,23)$, urbanismo ( $R \$ 344,53$ ) e cultura $(R \$ 30,64)$. Assim, após efetuada a estatística descritiva do modelo, testes foram 
efetuados para verificar a ocorrência de falha estrutural no modelo proposto, por meio do aplicativo Shapiro-Wilk, verificando a distribuição dos resíduos.

Conforme o resultado do teste $(p$-value $=0,477)$, pode-se afirmar que a distribuição dos resíduos é normal, assumindo que os testes do modelo de regressão são válidos. Após a realização do teste de normalidade, o pressuposto de normalidade foi aceito, tornando-se um indicador para verificar a heterocedasticidade do modelo. Dessa forma, para verificar a heterocedasticidade do modelo proposto, a qual pode ocorrer pela presença de dados discrepantes, erros de especificação, omissão de variáveis relevantes ou assimetria na distribuição de um ou mais dos regressores, realizou-se o teste White, conforme apresentado na Tabela 6:

Tabela 6

Teste White e Breusch-Pagan

\begin{tabular}{cc}
\hline Teste White & Teste Breusch-Pagan \\
\hline$L M=30,9317$ & $L M=8,309$ \\
\hline$p$-value $=0,273953$ & $p$-value $=0,140$ \\
\hline
\end{tabular}

Por meio dos testes White e Breusch-Pagan, pode-se inferir que, no modelo proposto, não há problemas com heterocedasticidade, ou seja, a variação do modelo é constante. Depois de realizados os testes de pressupostos para a regressão do modelo proposto, a Tabela 7 apresenta os resultados encontrados:

Tabela 7

Regressão linear

\begin{tabular}{lllll}
\hline \multicolumn{1}{c}{ Variáveis } & \multicolumn{1}{c}{ Coeficiente } & \multicolumn{1}{c}{ Erro padrão } & Razão-t & Sig. \\
\hline Constante & $6.619,731$ & $7.383,927$ & 0,897 & 0,378 \\
Saúde & 43,243 & 17,080 & 2,532 & $0,018^{* *}$ \\
Educação & 12,573 & 13,447 & 0,935 & 0,358 \\
Cultura & $-250,611$ & 145,362 & $-1,724$ & $0,097^{*}$ \\
Urbanismo & 28,738 & 30,673 & 0,937 & 0,357 \\
Investimento & $-2,194$ & 3,512 & $-0,625$ & 0,538 \\
R2 & 0,768 & R2 ajustado & 0,724 & \\
\hline
\end{tabular}

Nota. ${ }^{* * *}$ sig. $1 \%{ }^{* *}$ sig. $5 \%{ }^{*}$ sig. $10 \%$.

Observa-se na Tabela 7, por meio do $R^{2}(0,768)$ e $R^{2}$ ajustado $(0,724)$, que os gastos com saúde, educação, cultura, urbanismo e investimento possuem um forte poder de explicação em relação ao PIB per capita, o que denota associação entre os gastos e o PIB per capita das cidades portuárias. Assim, não se rejeita a hipótese de que as variáveis estudadas influenciam o PIB per capita de cidades portuárias, pois esses gastos explicam $76,8 \%$ do PIB dessas cidades. 
Com base nos dados da regressão linear, percebe-se que a variável saúde foi positiva e significativa a $5 \%$ ( $p$-value $=0,018)$, demostrando que, em média, $43,24 \%$ do PIB per capita dessas cidades corresponde à saúde, enquanto a cultura se apresentou de forma negativa $e$ significativa a $10 \%$ ( $p$-value $=0,097$ ), demonstrando que a cultura influencia o PIB per capita de forma negativa nas cidades portuárias. Assim, é possível inferir a significância da saúde em razão dos fatores negativos gerados pelos portos a essas cidades, tendo que ter um maior investimento em saúde para não afetar o bem-estar da população.

Percebe-se que a variável educação foi positiva e não significativa ( $p$-value=0,358), mas representa $12,57 \%$ do PIB per capita dessas cidades, já os gastos com urbanismo se apresentaram de forma positiva e não significativa ( $p$-value $=0,357$ ), demonstrando que a sua influência no PIB per capita das cidades portuárias é de $28,73 \%$.

De modo geral, os resultados mostraram que os gastos públicos se relacionam com o crescimento econômico das cidades portuárias, e que isso se mostra a partir do teste estatístico $R^{2}$, em que foi revelado que as variáveis que representaram os gastos públicos possuem forte poder de explicação em relação ao PIB per capita, com um poder de explicação de $76,8 \%$.

Os resultados adquiridos nesta pesquisa fazem coro com as pesquisas de Bogoni et al. (2011) e Merza e Alhasan (2016), e apesar do escopo distinto e da mesma simetria que foram conduzidas as pesquisas, é possível destacar certa harmonia neste trabalho. Com base nos resultados da pesquisa, Bogoni et al. (2011) concluíram que é relevante o papel do governo local no provimento dos serviços públicos, os quais afetam a produtividade, a qualidade de vida dos trabalhadores e, consequentemente, o crescimento econômico. Merza e Alhasan (2016), em suas pesquisas no Kuwait, concluíram que os gastos com saúde e educação também apresentam uma relação de equilíbrio de longo prazo com o PIB per capita.

Para Wagner (1883), o crescimento da renda per capita exige cada vez mais do governo a oferta de mais bens públicos. E, nesse sentido, os resultados decorrentes da aplicação do modelo estatístico nas 32 cidades portuárias corroboraram parcialmente com o estabelecido por Wagner, pois as variáveis saúde e investimento tiveram uma relação direta com o PIB per capita.

Entretanto, das três variáveis com relação direta, a saúde apresentou o maior percentual de significância, 5\%. De acordo com Barro (1990), essas variáveis representam os gastos produtivos, pois esses tipos de gastos geram externalidades positivas, que podem ser absorvidas por parte significativa da economia local, impulsionando o crescimento econômico.

Nesse contexto, tanto Bloom et al. (2004) quanto Mayer (2001) enfatizam a importância dos gastos públicos em saúde, na promoção do crescimento econômico. Na acepção de Thomas e Frankenberg (2002), a sociedade mais saudável proporciona uma população com maior expectativa de vida, acelerando a transição demográfica e estimulando o crescimento 
econômico. Para Bloom et al. (2004) e Mayer (2001), a falta de saúde correspondente à perda de horas de trabalho, com um declínio nas capacidades físicas e mentais dos trabalhadores, na produtividade e nos salários em geral.

Com objetivo distinto, mas em consonância com o estudo de Chan et al. (2017), os resultados foram convergentes e revelaram o seguinte: a eficiência dos gastos do governo promove o crescimento econômico; o sistema IVA aumenta o efeito da eficiência dos gastos do governo acerca do crescimento econômico; e a qualidade da democracia e força do Legislativo aumentaria ainda mais o papel moderador do sistema do IVA.

Contudo, os resultados do presente estudo encontraram discordância nos achados de Oliveira (2004), cujo papel das transferências de renda pelo governo local tem pouco ou quase nenhum efeito acerca do crescimento econômico e populacional das cidades nordestinas. Os gastos públicos são primordiais para o aprovisionamento de infraestruturas e bens públicos, que são capazes de produzir externalidades positivas, e estas podem ser absorvidas pelas empresas e, por consequência, aumentar a produtividade de toda a economia. Nesse sentido, os gastos (investimentos públicos locais) podem ser um determinante na atratividade de investimentos privados, o que refletiria positivamente no crescimento das cidades.

\section{CONCLUSÃO}

O estudo objetivou investigar a relação existente entre o crescimento econômico, considerando o PIB per capita e as variáveis que compõem os gastos públicos (saúde, educação, cultura, urbanismo e investimento) das cidades portuárias brasileiras. Com tal finalidade, o artigo apresenta um modelo estatístico, avaliado empiricamente por meio de uma regressão linear simples para os gastos públicos dessas cidades.

Com base na estatística descritiva, foi possível constatar que a média dos gastos públicos com saúde e educação nesses municípios é a maior, quando comparados com os demais gastos, podendo inferir que nas cidades portuárias há um maior investimento em saúde e educação por fatores advindos das condições originadas desses portos. Quanto ao desvio padrão amostral, percebe-se a maior variabilidade em relação à média amostral dos gastos com investimento, saúde e educação.

Por meio do $R^{2}(0,768)$, percebe-se que os gastos com saúde, educação, cultura, urbanismo e investimento possuem um forte poder de explicação em relação ao PIB per capita, o que denota associação entre os gastos e o PIB per capita das cidades portuárias. Tendo em vista os aspectos observados, pode-se dizer que as variáveis de gastos analisadas influenciam o crescimento econômico das cidades portuárias, representado pelo PIB per capita, com um poder de explicação de $76,8 \%$. 
Com base nos dados da regressão linear, percebe-se que a variável saúde foi positiva e significativa, demonstrando que em média $43,24 \%$ do crescimento econômico dessas cidades corresponde à saúde, enquanto a cultura influencia o crescimento econômico de forma negativa. Pode-se inferir essa significância da saúde em decorrência dos fatores negativos que os portos geram a essas cidades, tendo que ter um maior investimento em saúde para não afetar o bem-estar da população.

Os gastos com educação foram positivos e não significativos, representando $12,57 \%$ do crescimento econômico, enquanto os gastos com urbanismo se apresentaram de forma positiva e não significativa, demonstrando que influenciam o crescimento econômico das cidades portuárias em $28,73 \%$. Levando em consideração os aspectos analisados, pode-se concluir que os gastos com saúde, educação, cultura, investimento e urbanismo influenciam o crescimento econômico das cidades portuárias.

Este estudo apresenta limitações relacionadas aos recortes efetuados, pois os principais recortes se referem à amostra selecionada e ao ano analisados. No que diz respeito à amostra, os resultados da pesquisa se aplicam somente às cidades elencadas para este estudo (as cidades portuárias). De modo igual, o modelo estatístico somente foi testado para os gastos públicos efetuados pelas cidades em 2013, e o PIB per capita delas em 2015, porém houve uma defasagem de período pela não disponibilidade dos valores referentes ao PIB per capita com informação mais recente, datada de 2017, e também se justifica a utilização dos gastos no período de 2013 em razão do tempo necessário para a maturação dos gastos e investimentos realizados.

De modo complementar à proposta deste estudo, ressalta-se que é impossível obter informações de todos os elementos que se pretendem estudar, em decorrência da numerosidade de dados, da relação custo-benefício, da acessibilidade aos dados ou da limitação de tempo (Colauto \& Beuren, 2012).

Em razão dos resultados, concernentes à relação entre os gastos públicos e o crescimento econômico nas cidades portuárias, esta pesquisa gerou novos prognósticos e evidências empíricas acerca, particularmente, dos aspectos de políticas públicas das cidades portuárias. No entanto, o crescimento econômico pode estar além dos aspectos dos gastos públicos; uma abordagem de linha de base quádrupla é fundamental incluindo administração, economia, desenvolvimento econômico e gestão pública.

Por fim, recomenda-se que futuras pesquisas investiguem outros elementos, outras variáveis e recorte temporal maior, com informações apreendidas em uma temporalidade maior, possibilitando a sua precisão, e verifiquem a relação e o comportamento das variáveis em relação ao PIB per capita. Também poderiam analisar um modelo semelhante incluindo variáveis de controle como: classificação de cidades pelo tamanho dos portos e classificação das cidades por mesorregiões geográficas. 
Além das sugestões mencionadas, outros aspectos interessantes a serem analisados são: o desempenho financeiro dos portos e seus reflexos nas receitas das cidades portuárias; investigar os gastos públicos e a relação da governança nos governos locais das cidades portuárias, conforme estudo realizado por Rajkumar e Swaroop (2008).

\section{REFERÊNCIAS}

Acciaro, M. (2008). The role of ports in the development of Mediterranean islands: The case of Sardinia. International Journal of Transport Economics/Rivista internazionale di economia dei trasporti, 295-323.

Al-Faris, A. F. (2002). Public expenditure and economic growth in the Gulf Cooperation Council countries. Applied economics, 34(9), 1187-1193.

Araújo, E., Fajardo, J., \& Tavani, L. C. D. (2006). CAPM usando uma carteira sintética do PIB Brasileiro. Estudos Econômicos, 36(3), 465-505.

Ashwani, B., \& Sheera, V. P. (2017). Public spending and economic growth for Indian states. International Journal of Public Sector Performance Management, 3(3), 250-265.

Barro, R. J. (1990, October). Government spending in a simple model of endogenous growth. Journal of Political Economy, 98, 103-125.

Bloom, D. E., Canning, D., \& Sevilla, J. (2004). The effect of health on economic growth: A production function approach. World development, 32(1), 1-13.

Bogoni, N. M., Hein, N., \& Beuren, I. M. (2011, janeiro/fevereiro). Análise da relação entre crescimento econômico e gastos públicos nas maiores cidades da Região Sul do Brasil. Revista de Administração Pública, 45(1), 159-179.

Bottasso, A., Conti, M., Ferrari, C., Merk, O., \& Tei, A. (2013). O impacto da produção portuária no emprego local: Evidências de um painel de regiões europeias. Política de Transporte, 27, 32-38.

Castro, C. M. (1977). A prática da pesquisa. Estados Unidos: McGraw-Hill.

Chan, S. G., Ramly, Z., \& Karim, M. Z. A. (2017). Government Spending Efficiency on Economic Growth: Roles of Value-added Tax. Global Economic Review, 46(2), 162-188. 
Chen, B. L. (2006). Economic growth with an optimal public spending composition. Oxford Economic Papers, 58(1), 123-136.

Colauto, R. D., Beuren, I. M. (2012). Coleta, Análise e Interpretação dos dados. In I. M. Beuren (Org.), Como elaborar trabalhos monográficos em contabilidade: Teoria e prática (pp. 117-144). São Paulo: Atlas.

Cooper, D. R., \& Schindler, P. S. (2011). Métodos de pesquisa em administração. (10 ed.). Porto Alegre: Bookman.

Ensslin, L., Dezem, V., Dutra, A., Ensslin, S. R., \& Somensi, K. (2017). Seaport-performance tools: an analysis of the international literature. Maritime Economics \& Logistics, 1, 1-16.

Ensslin, S. R., Dutra, A., Ensslin, L., Valmorbida, S. M. I., \& Cardoso, T. L. (2015). Avaliação Multicritério de Desempenho de Portos Marítimos Brasileiros: Estudo de Caso para apoiar a gestão do Porto São Francisco do Sul. Anais do Cidesport - Congresso Internacional de Desempenho Portuário, Florianópolis, 2, 1-25.

Ferreira, P. C., \& Malliagros, T. G. (1998). Impactos produtivos da infra-estrutura no Brasil 1950/95. Pesquisa e Planejamento Econômico, 28(2), 315-338.

Ferreira, P. C. (1996). Investimento em infra-estrutura no Brasil: Fatos estilizados e relações de longo prazo. Pesquisa e Planejamento Econômico, 26(2), 231-252.

Furtuoso, M. C. O., \& Guilhoto, J. J. M. (2003). Estimativa e mensuração do Produto Interno Bruto do Agronegócio da Economia Brasileira, 1994 a 2000. Revista de Economia e Sociologia Rural, 41(4), 803-827.

Giner-Fillol, A., Lunkes, R. J., Ripoll-Feliu, V. M., \& Rosa, F. S. (2013). Gestão Portuária - Com caso prático no Porto de Valência (Valencia Port). Insular, 192.

Guerrero, J. I. F., Selva, L. M., \& Medina, R. P. (2008). Economic impact of Western Mediterranean leisure ports. International Journal of Transport Economics/Rivista internazionale di economia dei trasporti, 251-272.

Gyimah-Brempong, K., \& Wilson, M. (2004). Health human capital and economic growth in Sub-Saharan African and OECD countries. The Quarterly Review of Economics and Finance, 44(2), 296-320.

Hsieh, E., \& Lai, K. S. (1994). Government spending and economic growth: the G-7 experience. Applied Economics, 26(5), 535-542. 
Instituto Brasileiro de Geografia e Estatística. (2017). Recuperado de www.ibge.gov.br

Irmen, A., \& Kuehnel, J. (2009). Productive government expenditure and economic growth. Journal of Economic Surveys, 23(4), 692-733.

Jouili, T., \& Allouche, M. A. (2016). Investment in seaports and economic growth. International Journal of Transport Economics. Rivista Internazionale de Economia dei Trasporti, $43(4)$.

Kolluri, B. R., Panik, M. J., \& Wahab, M. S. (2000). Government expenditure and economic growth: Evidence from G7 countries. Applied Economics, 32(8), 1059-1068.

Landau, D. (1983). Government expenditure and economic growth: A cross-country study. Southern Economic Journal, 783-792.

Lea, T. M. H., Choib, H. R., \& Alfaroc, L. A. M. (2017). An Exploratory Study on Policy of the Public-Private Partnership to Develop Seaport in Vietnam. International Journal of Applied Business and Economic Research, 15(3), 57-68.

Lupu, D., \& Asandului, M. (2017). The Nexus between Economic Growth and Public Spending in Eastern European Countries. Engineering Economics, 28(2), 155-161.

Mayer, D. (2001). The long-term impact of health on economic growth in Latin America. World development, 29(6), 1025-1033.

Merza, E., \& Alhasan, N. (2016). Public Spending and Economic Growth in the Rentier State: The Case of Kuwait. Asian Social Science, 12(8), 160.

Ministério dos Transportes, Portos e Aviação Civil. (2017). Lista de Portos Nacionais. Recuperado de www.transporte.gov.br

Monié, F., \& Vidal, S. M. do S. C. (2006). Cidades, portos e cidades portuárias na era da integração produtiva. Revista de Administração Pública - RAP, 4O(6).

Morozumi, A., \& Veiga, F. J. (2016). Public spending and growth: The role of government accountability. European Economic Review, 89, 148-171.

Ogundari, K., \& Abdulai, A. (2014). Determinants of household's education and healthcare spending in Nigeria: Evidence from survey data. African Development Review, 26(1), 1-14. 
Ogundari, K., \& Awokuse, T. (2018). Human capital contribution to economic growth in Sub-Saharan Africa: Does health status matter more than education? Economic Analysis and Policy, 58, 131-140.

Oliveira, C. A. (2004). Crescimento econômico das cidades nordestinas: Um enfoque da nova geografia econômica. Anais do Encontro Regional de Economia, Fortaleza, 9.

Pegkas, P., \& Tsamadias, C. (2014). Does higher education affect economic growth? The case of Greece. International Economic Journal, 28(3), 425-444.

Rajkumar, A. S., \& Swaroop, V. (2008). Public spending and outcomes: Does governance matter? Journal of development economics, 86(1), 96-111.

Riani, F. (2002). Economia do Setor Público: Uma abordagem introdutória. (4a ed.) São Paulo: Atlas.

Richardson, R. J. (1999). (1999). Pesquisa Social: Métodos e Técnicas. (3a ed.). São Paulo: Atlas.

Samudram, M., Nair, M., \& Vaithilingam, S. (2009). Keynes and Wagner on government expenditures and economic development: The case of a developing economy. Empirical Economics, 36(3), 697-712.

Secretaria do Tesouro Nacional. (2017). Contabilidade governamental. Recuperado de www. stn.gov.br

Shan, J., Yu, M., \& Lee, C. Y. (2014). An empirical investigation of the seaport's economic impact: Evidence from major ports in China. Transportation Research Part E: Logistics and Transportation Review, 69, 41-53.

Sinha, D. (1998). Government expenditure and economic growth in Malaysia. Journal of Economic Development, 23(2), 71-80.

Song, L., \& Mi, J. (2016). Port infrastructure and regional economic growth in China: A Granger causality analysis. Maritime Policy \& Management, 43(4), 456-468.

Thomas, D., \& Frankenberg, E. (2002). Health, nutrition and prosperity: A microeconomic perspective. Bulletin of the World Health Organization, 80, 106-113.

Tukan, M., Achmadi, T., \& Widjaja, S. (2015). Seaport Dimensional Analysis towards Economic Growth in Archipelagic Regions. International Journal of Technology, 6(3), 422-431. 
Van Niekerk, H. C. (2005). Port reform and concessioning in developing countries. Maritime Economics \& Logistics, 7(2), 141-155.

Zanmaria, N. A., \& Castilho, M. L. (2006). Gastos públicos com educação e seus reflexos no crescimento econômico brasileiro. Revista Faz Ciência, 8(1), 123.

\section{Como citar este artigo:}

\section{ABNT}

SILVA, Cristiane Aparecida da et al. Análise entre os gastos do governo local e o crescimento econômico das cidades portuárias. RACE, Revista de Administração, Contabilidade e Economia, Joaçaba: Ed. Unoesc, v. 18, n. 1, p. 177-200, jan./abr. 2019. Disponível em: https://portalperiodicos.unoesc.edu.br/race. Acesso em: dia/mês/ano.

\section{APA}

Silva, C. A. da, Amaral, B. G., Anzilago, M., \& Lunkes, R. J. (2019). Análise entre os gastos do governo local e o crescimento econômico das cidades portuárias. RACE, Revista de Administração, Contabilidade e Economia, 18(1), 177-200 Recuperado de http://editora. unoesc.edu.br/index.php/race 
